Curtis T. McMullen

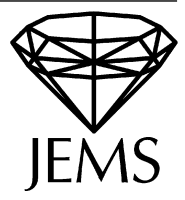

\title{
Navigating moduli space with complex twists
}

Received December 5, 2011 and in revised form August 22, 2012 and September 19, 2012

\begin{abstract}
We discuss a common framework for studying twists of Riemann surfaces coming from earthquakes, Teichmüller theory and Schiffer variations, and use it to analyze geodesics in the moduli space of isoperiodic 1-forms.
\end{abstract}

\section{Introduction}

The moduli space of Riemann surfaces can be navigated using Fenchel-Nielsen twists, earthquakes, grafting, the Teichmüller geodesic flow, Schiffer variations and various other geometric constructions.

In this paper we give a common framework for all these operations, and apply it to study the moduli space of holomorphic 1-forms, $\Omega \mathcal{M}_{g} \rightarrow \mathcal{M}_{g}$. This moduli space has a flat metric along the leaves of its absolute period foliation $\mathcal{A}$. By relating Schiffer variations to complex twists, we show that geodesics along the leaves of $\mathcal{A}$ behave well geometrically: they are either complete, or they terminate via a collision of zeros or the creation of a node.

Twists. Let $X$ be a Riemann surface. We begin in $\$ 2$ by defining the complex twist $\delta(C, v)$, a tangent vector to moduli space at $X$ specified by a holomorphic vector field $v$ along an oriented simple loop $C \subset X$. Cotangent vectors to moduli space are specified by integrable holomorphic quadratic differentials $q \in Q(X)$, and we find:

Theorem 1.1. The natural pairing between a complex twist and a quadratic differential is given by

$$
\langle\delta(C, v), q\rangle=\frac{i}{2} \int_{C} q v .
$$

As a special case we recover Wolpert's formula for the Fenchel-Nielsen twist [Wol1, Lemma 4.2]. More generally, for an earthquake along a lamination $\lambda$ with transverse measure $m$ we find

$$
\langle\operatorname{tw}(\lambda), q\rangle=\frac{i}{2} \int_{\lambda} q v^{2}|d s| d m .
$$

Here $v$ is a unit vector field tangent to $\lambda$ and $d s$ denotes arclength in the hyperbolic metric.

C. T. McMullen: Mathematics Department, Harvard University, 1 Oxford St, Cambridge, MA 02138-2901, USA 
As another example, let $C_{p}$ be a small loop around $p \in X$ and choose local coordinates with $z(p)=0$. We will see the Schiffer variation at $p$ is given by

$$
\mu_{p}=\delta(C,-1 / z)
$$

which implies Gardiner's formula $\left\langle\mu_{p}, q\right\rangle=\pi q(0)$ for all $q \in Q(X)$ (see [Ga, §5]). Here we have used the preferred local coordinate $z$ to write $q=q(z) d z^{2}$.

Moduli of 1-forms. In $\S 3$ we turn to the moduli space of holomorphic 1-forms,

$$
\pi: \Omega \mathcal{M}_{g} \rightarrow \mathcal{M}_{g}
$$

an orbifold bundle whose fiber over $X$ consists of the nonzero holomorphic 1-forms $\omega \in \Omega(X)$ modulo the action of the finite group $\operatorname{Aut}(X)$. This space has many interesting features; for example, it carries a natural action of $\mathrm{SL}_{2}(\mathbb{R})$ with rich topological and measurable dynamics, which in turn are related to polygon billiards (see e.g. [D]).

The moduli space of 1-forms is partitioned into strata $\Omega \mathcal{M}_{g}\left(m_{i}\right)$ according to the multiplicities of the zeros of $\omega$. The tangent space to a stratum at $(X, \omega)$ is naturally identified with the complex cohomology group $H^{1}(X, Z(\omega))$, where $Z(\omega)$ is the zero set of $\omega$. We begin by computing the derivative of the projection

$$
\pi: \Omega \mathcal{M}_{g}\left(m_{i}\right) \rightarrow \mathcal{M}_{g}
$$

Theorem 1.2. The projection of a tangent vector $\phi \in H^{1}(X, Z(\omega))$ to moduli space is given by the complex twist

$$
D \pi(\phi)=\delta(C, 1 / \omega),
$$

where $C \in H_{1}(X-Z(\omega))$ is the unique cycle satisfying $\phi(E)=C \cdot E$ for all $E \in$ $H_{1}(X, Z(\omega))$.

Here $\delta(C, 1 / \omega)$ is extended by linearity to complex homology classes.

Twists of 1-forms. Let $\operatorname{tw}(C)$ denote the tangent vector to $\Omega \mathcal{M}_{g}$ represented by a cycle $[C] \in H_{1}(X-Z(\omega))$, as above. When $C$ is a simple loop, $\operatorname{tw}(C)$ is given geometrically by cutting $(X, \omega)$ open along $C$, shifting horizontally at unit speed in coordinates where $\omega=d z$, and regluing. If $C$ happens to be a horizontal geodesic for the metric $|\omega|$, then $\operatorname{tw}(C)$ is simply a flat version of the Fenchel-Nielsen twist.

Foliations. The absolute periods of $\omega$ define a local submersion of orbifolds

$$
\Omega \mathcal{M}_{g} \supset U \rightarrow H^{1}(X) / \operatorname{Aut}(X),
$$

whose fibers are the leaves of the absolute period foliation $\mathcal{A}$. This foliation is transverse to the fibers of $\pi$ and to the orbits of $\mathrm{SL}_{2}(\mathbb{R})$. Its restriction to a stratum will be denoted by $\mathcal{A}\left(m_{i}\right)$. 
Metrics along the leaves. It is easy to see that the tangent space to $\mathcal{A}\left(m_{i}\right)$ at $(X, \omega)$ is generated by twists around small loops $C_{p}$ encircling the zeros of $\omega$, subject to the relation $\sum_{p \in Z(\omega)} \operatorname{tw}\left(C_{p}\right)=0$. A useful norm on these relative period deformations is given by

$$
\left\|\sum a_{p} \operatorname{tw}\left(C_{p}\right)\right\|_{R}=\max \left|a_{p}-a_{q}\right| .
$$

Schiffer variations. By (1.1), a twist around $C_{p}$ comes from a Schiffer variation. If $\omega \in \Omega(X)$ has a simple zero at $p$, this variation can be carried out geometrically by slitting $(X, \omega)$ open along the horizontal and vertical leaves through $p$, then folding and regluing to obtain a new 1 -form $\left(X^{\prime}, \omega^{\prime}\right)$ (see Figure 4 in $\S 3$ ). This folding operation is very useful in the study of 1 -forms, since it patently preserves not only the absolute periods, but also the geometry of $(X, \omega)$ away from the slit. In this way it can be compared to a Fenchel-Nielsen twist along a geodesic $C$, which preserves the hyperbolic metric on $X$ away from $C$.

The Schiffer folding operation can be carried out simultaneously at different zeros, so long as the slits remain disjoint, which shows:

Theorem 1.3. The leaf of $\mathcal{A}\left(m_{i}\right)$ through $(X, \omega)$ contains an immersed ball of radius $r$, where $2 r$ is the length of the shortest saddle connection on $(X, \omega)$.

Completeness. Now let $\gamma:[0, T) \rightarrow \Omega \mathcal{M}_{g}$ be a smooth path along a leaf of $\mathcal{A}\left(m_{i}\right)$ with $\left\|\gamma^{\prime}(t)\right\|_{R} \leq 1$. We would like to show that $\gamma(t)=\left(X_{t}, \omega_{t}\right)$ converges, as $t \rightarrow T$, to a suitable form $(Y, \eta)$ on a stable curve $Y \in \overline{\mathcal{M}_{g}}$.

This is not quite true; in fact, in $\S 4$ we will show:

The Riemann surface $\left[X_{t}\right]$ can oscillate and fail to have a limit in $\overline{\mathcal{M}_{g}}$ as $t \rightarrow T$.

However, this oscillation is eliminated if we form a collapsed moduli space $\Omega \overline{\mathcal{M}_{g}} / \sim$ by forgetting the components of $(Y, \eta)$ where $\eta$ vanishes identically. We then find:

Theorem 1.4. The limit $(Y, \eta)=\lim _{t \rightarrow T}\left(X_{t}, \omega_{t}\right)$ exists in $\Omega \overline{\mathcal{M}_{g}} / \sim$, and there is no loss of mass: $\int_{X_{t}}\left|\omega_{t}\right|^{2} \rightarrow \int_{Y}|\eta|^{2}$.

Now suppose $\gamma$ is also a relative period geodesic, meaning its direction $\gamma^{\prime}(t) \in$ $H^{1}(X, Z(\omega))$ is locally constant. Then $\gamma$ can be continued past $t=T$ unless $Y \notin \mathcal{M}_{g}$ or $(Y, \eta)$ is in a different stratum. This shows:

Corollary 1.5. A relative period geodesic in a stratum is either complete, or it terminates via a collision of zeros or the creation of a node.

The proof of Theorem 1.4 uses universal bounds for cylinders, given in the Appendix, to show no mass of $\omega_{t}$ can escape to a node of $Y$.

Application: Hilbert modular surfaces. The absolute period foliation and the natural metric along its leaves have been studied, for eigenforms of genus two, in [Mc3] and [Ba].

Let $\mathcal{O}_{D}$ denote the real quadratic order of discriminant $D>0$. Let

$$
\Omega \overline{E_{D}} \subset \Omega \overline{\mathcal{M}_{2}}
$$


denote the space of finite mass stable eigenforms $(Y, \eta)$ for real multiplication by $\mathcal{O}_{D}$. This locus is saturated by the leaves of $\mathcal{A}$ (cf. [Mc2, Cor. 5.6]). The mass condition implies $Y$ is either a smooth curve of genus 2 or a pair of curves of genus 1 joined at a node. In either case, $\operatorname{Jac}(Y)$ is compact.

Every principally polarized Abelian variety with real multiplication by $\mathcal{O}_{D}$ arises as such a Jacobian, so $\Omega \overline{E_{D}}$ forms a $\mathbb{C}^{*}$ bundle over the Hilbert modular surface

$$
X_{D}=(\mathbb{H} \times \mathbb{H}) / \operatorname{SL}\left(\mathcal{O}_{D} \oplus \mathcal{O}_{D}^{\vee}\right) .
$$

The points of $X_{D}$ correspond to eigenforms $(Y,[\eta])$ up to scale. On the universal cover of $X_{D}$, we can normalize at $\left(s, s^{\prime}\right)$ so the absolute periods of $\eta$ are given by $\mathcal{O}_{D} \oplus \mathcal{O}_{D}^{\vee} s$. The result is a map

$$
p: \mathbb{H} \times \mathbb{H} \rightarrow \Omega \overline{E_{D}}
$$

commuting with projection to $X_{D}$.

The absolute periods of $(Y, \eta)=p\left(s, s^{\prime}\right)$ are constant along any slice $\mathbb{H}_{s}=\{s\} \times \mathbb{H}$. The remaining relative period, given locally by

$$
\rho(Y, \eta)=\int_{p_{1}}^{p_{2}} \eta
$$

where $Z(\eta)=\left\{p_{1}, p_{2}\right\}$, determines a globally well-defined meromorphic quadratic differential $q=(d \rho)^{2}$ on $\mathbb{H}_{s}$. This differential has simple poles where $Y$ is singular, and simple zeros where $p_{1}=p_{2}$ (see $[\mathrm{Mc} 3, \S 8]$ ).

Using Theorem 1.4, we can now show:

Theorem 1.6. Any slice $\mathbb{H}_{S}$ of the universal cover of $X_{D}$ is complete in the metric $|q|$ determined by the relative period differential.

Proof. Let $\gamma:[0, T) \rightarrow \mathbb{H}_{s}$ be a path of finite length in the metric $|q|$. Consider the diagram

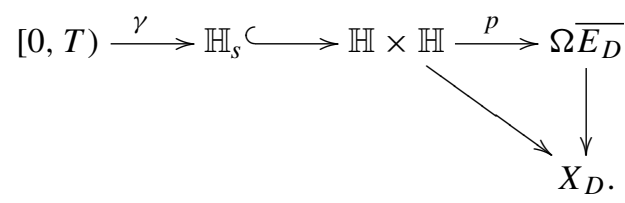

We wish to show $\lim _{t \rightarrow T} \gamma(t)$ exists in $\mathbb{H}_{s}$. To this end, first perturb $\gamma$ slightly so it avoids the zeros and poles of $q$. Then $p(\gamma(t))$ takes values in $\Omega E_{D}(1,1)$. Since $s$ is fixed, its image lies on a single leaf of $\mathcal{A}(1,1)$. Since $\int_{\gamma}|q|$ is finite, this path also has finite length in the relative period metric (1.2). So by Theorem 1.4, $p(\gamma(t))$ converges as $t \rightarrow T$ to a point $[(Y, \eta)] \in \Omega \overline{\mathcal{M}_{2}} / \sim$. Since we have not changed the absolute periods, we have $(Y, \eta) \in \Omega \overline{E_{D}}$. But an eigenform always has full support, so in fact $p(\gamma(t)) \rightarrow(Y, \eta) \in \Omega \overline{E_{D}}$. Projecting to $X_{D}$ and then lifting to its universal cover, we conclude that the corresponding paths $[0, T) \rightarrow X_{D}$ and $[0, T) \rightarrow \mathbb{H} \times \mathbb{H}$ also converge. But $\mathbb{H}_{S}$ is closed in $\mathbb{H} \times \mathbb{H}$, so the original path $\gamma:[0, T) \rightarrow \mathbb{H}_{S}$ converges as well. 
Notes and references. A general completeness result for the leaves of $\mathcal{A}\left(m_{i}\right)$ (with suitable stable forms adjoined) is established in a sequel to this paper [Mc4], which also treats the case of genus two in detail. The question of completeness was raised by Hooper, Smillie and Weiss.

For useful perspectives on the moduli spaces we consider here and the degeneration of Riemann surfaces, see e.g. [KZ], [Mas], [Mo], [Wol3] and [ACG].

Notation. The notation $A \asymp B$ means $A / B$ and $B / A$ are both bounded above by an implicit constant $C$. Absolute and relative cohomology groups with coefficients in $\mathbb{C}$ will be denoted by $H^{*}(X)$ and $H^{*}(X, Z)$ respectively; sheaf cohomology groups will be denoted $H^{*}(X ; \mathcal{F})$.

\section{Complex twists}

In this section we define the complex twist deformation $\delta(C, v)$ and show:

Theorem 2.1. For any holomorphic quadratic differential $q \in Q(X)$ we have

$$
\langle\delta(C, v), q\rangle=\frac{i}{2} \int_{C} q v .
$$

Note that $q v$ is naturally a holomorphic 1 -form, and the integral above gives its period around $C$. Thus $\delta(C, v)$ only depends on the homology class of $C$ in the domain of $v$.

We will show several other deformations are special cases of the complex twist, and obtain estimates for their size in the Teichmüller metric. For related discussions, see [Wol2] and [SS, §7.8].

Teichmüller theory. Let $X$ be any Riemann surface. Let $Q(X)$ and $M(X)$ denote the spaces of holomorphic quadratic differentials $q$ and measurable Beltrami differentials $\mu$ on $X$, respectively. We require that $\|q\|_{1}=\int_{X}|q|<\infty$ and $\|\mu\|_{\infty}<\infty$; then we have a natural pairing

$$
\langle\mu, q\rangle=\int_{X} \mu q,
$$

making $M(X) / Q(X)^{\perp}$ into the dual of $Q(X)$.

The Teichmüller space of $X$ parameterizes marked Riemann surfaces quasiconformally equivalent to $X$. Its tangent space at $X$ is naturally identified with $M(X) / Q(X)^{\perp}$, and the quotient norm

$$
\|\mu\|_{T}=\sup \left\{|\langle\mu, q\rangle|: q \in Q(X),\|q\|_{1}=1\right\}
$$

gives the Teichmüller metric.

Definition of a complex twist. Let $C$ be a smooth closed loop on a Riemann surface $X$ and $v$ a holomorphic vector field defined near $C$. Let $\Theta$ be the sheaf of holomorphic vector fields on $X$. We will define the deformation $\delta(C, v)$ as a (suitably bounded) element of the Čech cohomology group $H^{1}(X ; \Theta)$. 

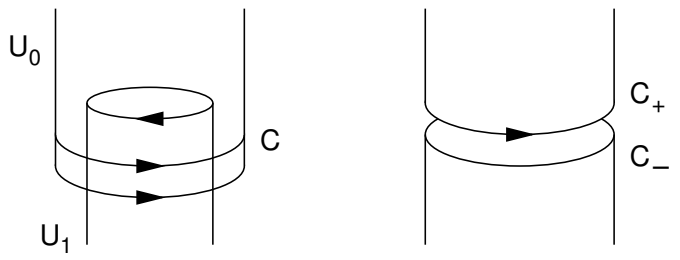

Fig. 1. Orientations.

To do this, choose an open covering $\left(U_{i}\right)$ of $X$ such that $A=U_{0} \cap U_{1}$ is an annular neighborhood of $C$ on which $v$ is defined, and $U_{0} \cap U_{1} \cap U_{j}=\emptyset$ for $j>1$. Assuming $\partial U_{i}$ is smooth and with the induced orientations, we have

$$
\left[A \cap \partial U_{0}\right]=-\left[A \cap \partial U_{1}\right]=[C] \quad \text { in } H_{1}(A) .
$$

(See Figure 1 (left).) We then define

$$
\left[v_{i j}\right] \in H^{1}(X ; \Theta)
$$

by $v_{01}=v \in \Theta(A), v_{10}=-v_{01}$, and $v_{i j}=0$ in all other cases. Note that $\delta(C, v)$ is a complex linear function of $v \in \Theta(A)$, and $\delta(-C,-v)=\delta(C, v)$.

The cocycle $\left[v_{i j}\right]$ determines a family of Riemann surfaces $X_{t}$ for small $t$ by gluing $z \in U_{i}$ to $z+t v_{i j}+O\left(t^{2}\right) \in U_{j}$. Hence it determines a tangent vector

$$
\delta(C, v)=\left.\frac{d X_{t}}{d t}\right|_{t=0}
$$

to Teichmüller space at $X$. (In fact, when $X$ is compact, we have a natural isomorphism $H^{1}(X ; \Theta) \cong M(X) / Q(X)^{\perp}$.)

Proof of Theorem 2.1. Let $\phi$ be a compactly supported smooth function on $\overline{U_{1}}$, equal to one on $A$ and vanishing outside the domain of $v$. Then $w_{1}=\phi v$ extended by zero is a smooth vector field on $U_{1}$. Defining $w_{i}=0$ for $i \neq 1$, we have

$$
v_{i j}=w_{j}-w_{i}
$$

for all $i, j$. In other words, $\left[v_{i j}\right]$ is the coboundary of the 0 -cycle $\left[w_{i}\right]$. Geometrically, this means for small $t$ we have a smooth map $f_{t}: X_{0} \rightarrow X_{t}$ given by $f_{t}(z)=z+t w_{i}+O\left(t^{2}\right)$ on $U_{i}$. Condition (2.4) ensures that the diagram

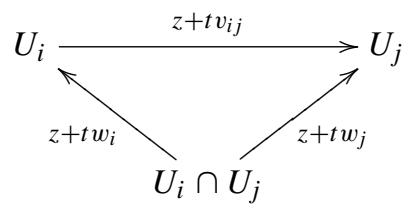

commutes to first order in $t$. 
Since $w_{j}-w_{i}$ is holomorphic, there is a unique Beltrami differential $\mu \in M(X)$ such that $\mu=\bar{\partial} w_{i}$ on $U_{i}$. This $\mu$ represents the tangent vector $\delta(C, v)$ to $X$ in Teichmüller space. To complete the proof, we now compute:

$$
\begin{aligned}
\langle\delta(C, v), q\rangle & =\int_{X} q \mu=\int_{U_{1}} q \bar{\partial}(\phi v)=\frac{i}{2} \int_{U_{1}} q(z) v(z) d z \wedge d \phi \\
& =-\frac{i}{2} \int_{U_{1}} d(\phi q v)=-\frac{i}{2} \int_{\partial U_{1}} q v=\frac{i}{2} \int_{C} q v .
\end{aligned}
$$

The factor $i / 2$ arises from replacing $|d z|^{2}$ with $(i / 2) d z \wedge d \bar{z}$, and the last change of sign comes from (2.3).

Real twisting. Assume $v$ is tangent to $C$ and nowhere vanishing. In this case $C$ is a real analytic curve, and we can use $v$ to twist along $C$ for any time $t \in \mathbb{R}$ to obtain a new Riemann surface $X_{t}$.

Indeed, the tangency assumption implies there is a real-analytic flow $f_{t}: C \rightarrow C$ satisfying $f_{0}(z)=z$ and $d f_{t} / d t=v$. Cutting $X$ open along $C$, we obtain a pair of curves $C_{+} \cong C \cong C_{-}$, where the induced orientation of $C_{+}$agrees with that of $C$ (see Figure 1 (right)). To obtain $X_{t}$, we simply reglue using the map $f_{t}: C_{+} \rightarrow C_{-}$. The direction of regluing is chosen so that

$$
\delta(C, v)=\left.\frac{d X_{t}}{d t}\right|_{t=0} .
$$

Fenchel-Nielsen. Now suppose $C$ is an unoriented closed geodesic on a hyperbolic Riemann surface $C$. Let $v$ be a continuous vector field of length 1 tangent to $C$, and orient $C$ in the same direction as $v$. Then $v$ locally extends to a holomorphic vector field, and we obtain the Fenchel-Nielsen twist deformation

$$
\operatorname{tw}(C)=\delta(C, v) .
$$

It gives a right twist at unit speed along $C$ that can be defined for all time. By the results above, we have

$$
\langle\operatorname{tw}(C), q\rangle=\frac{i}{2} \int_{C} q v^{2}|d s| .
$$

Here $q v^{2}$ is naturally a function, and $|d s|$ denotes arclength along $C$. Compare [Wol1, Lemma 4.2].

Earthquakes. Since the formula above no longer involves the orientation of $C$, we can pass to the limit and obtain a similar result for the tangent vector defined by a right earthquake along a measured lamination $\lambda$ on $X$ [Ker], [Th], [GL, §18]. Namely, we have

$$
\langle\operatorname{tw}(\lambda), q\rangle=\frac{i}{2} \int_{\lambda} q v^{2}|d s| d m,
$$

where $d m$ denotes the transverse measure to $\lambda$, and $v$ is locally a unit vector field tangent to $\lambda$. 
Grafting. One can also cut open along $C$ and insert a Euclidean annulus of width $t$. The resulting grafting deformation is given by $\operatorname{gr}(C)=\delta(C, i v)=i \delta(C, v)$. The combination of twisting and grafting yields complex earthquakes disks in Teichmüller space [Mc1].

Twisting quadratic differentials. A quadratic differential $q_{0}$ determines a pair of vertical and horizontal measured foliations on $X$. In a local chart where $q_{0}=d z^{2}$, their leaves are vertical and horizontal lines.

Let $\operatorname{tw}\left(C, q_{0}\right)$ denote a unit speed right twist on a smooth closed leaf of the horizontal foliation of $q_{0}$, with respect to the metric $\left|q_{0}\right|^{1 / 2}$. We then have

$$
\left\langle\operatorname{tw}\left(C, q_{0}\right), q\right\rangle=\delta(C, v)=\frac{i}{2} \int_{C} \frac{q}{q_{0}}\left|q_{0}\right|^{1 / 2} .
$$

This equation is parallel to (2.6) with $v^{2}=1 / q_{0}$.

As in the case of an earthquake, we can also consider twisting along the horizontal measured foliation $\mathcal{F}$. This is equivalent to the shearing deformation $f_{t}: z \mapsto z+t \operatorname{Im}(z)$ in local coordinates where $q_{0}=d z^{2}$, and we find

$$
\left\langle\operatorname{tw}\left(\mathcal{F}, q_{0}\right), q\right\rangle=\frac{i}{2} \int_{X} \frac{q}{q_{0}}\left|q_{0}\right| .
$$

This is analogous to the earthquake formula (2.7), with $|d s| d m=|d q|$. As a consistency check, note that the Beltrami coefficient

$$
\mu=\bar{\partial}(d f / d t)=\bar{\partial}(\operatorname{Im}(z) d / d z)=(i / 2) d \bar{z} / d z=(i / 2)\left|q_{0}\right| / q_{0}
$$

represents the same deformation as $\operatorname{tw}\left(\mathcal{F}, q_{0}\right)$, and that the integral above coincides with the usual pairing between $\mu$ and $q$.

For vertical closed leaves and for the vertical foliation, the corresponding formulas acquire a minus sign.

The Teichmüller flow. A quadratic differential $q_{0}$ also determines a unit speed Teichmüller geodesic $X_{t}$. The extremal map $f_{t}: X_{0} \rightarrow X_{t}$ has the form

$$
f_{t}(x+i y)=e^{t} x+i e^{-t} y
$$

in local coordinates where $q_{0}=d z^{2}, z=x+i y$, and thus $[\mu]=d X_{t} /\left.d t\right|_{t=0}=\left|q_{0}\right| / q_{0}$.

Note that $[\mu]=-2 i \operatorname{tw}\left(\mathcal{F}, q_{0}\right)$. Thus $X_{t}$ is obtained by grafting along the vertical foliation of $q_{0}$.

The Schiffer variation. We now turn to a folding deformation supported near a single point $p \in X$, introduced by Schiffer [Sch], [Ah, §7], [IT, §A].

Let $\omega$ be a locally defined holomorphic 1-form on $X$ with a zero at $p$. Choose local coordinates so that $z(p)=0$ and $\omega=(z / 2) d z$. For small $t \geq 0$, let $A_{t}=i[-2 u, 2 u] \subset X$ denote a vertical arc in these coordinates with $u^{2}=t$. Then $\int_{A_{t}}|\omega|=2 t$. Slit $X$ open along $A_{t}$, and fold each of the two resulting arcs so that $z$ is identified with $-z$. (For example, $2 i u$ is glued to $-2 i u$; see Figure 2.) 


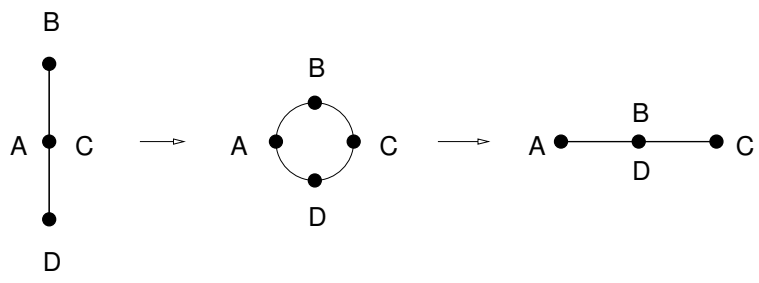

Fig. 2. The Schiffer variation.

The result is a new Riemann surface $X_{t}$ with a horizontal $\operatorname{arc} B_{t}$, and a natural holomorphic map $f_{t}: X-A_{t} \rightarrow X_{t}-B_{t}$. More concretely, in local coordinates on $X_{t}$ we can write $f_{t}(z)=g_{+} \circ g_{-}^{-1}(z)$, where

$$
g_{ \pm}(z)=z \pm \frac{t}{z}
$$

The images of the circle $|z|=u$ under $g_{-}$and $g_{+}$are then $A_{t}$ and $B_{t}=[-2 u, 2 u]$ respectively. (The normalization $\omega=(z / 2) d z$ was chosen to put $g_{ \pm}(z)$ into this simple form.)

Since $g_{-}^{*}(z d z)=g_{+}^{*}(z d z)$, there is a natural holomorphic 1-form $\omega_{t}$ defined near $B_{t}$ such that

$$
f_{t}^{*} \omega_{t}=\omega
$$

If $\omega$ is globally defined, so is $\omega_{t}$.

The Schiffer variation of $X$ is defined by $\operatorname{Sch}(\omega, p)=d X_{t} /\left.d t\right|_{t=0}$.

Theorem 2.2. The Schiffer variation satisfies $\operatorname{Sch}(\omega, p)=\delta(C,-1 / \omega)$ for any small, positively oriented loop around $p$. Thus

$$
\langle\operatorname{Sch}(\omega, p), q\rangle=\pi \operatorname{Res}_{p}(q / \omega)
$$

for any $q \in Q(X)$.

Proof. Let $X=U_{0} \cup U_{1}$ be an open cover such that $U_{0}$ is a small disk containing $p$ and $C$, and $U_{0} \cap U_{1}$ is an annular neighborhood of $C$ disjoint from $p$. Then $X_{t}$ is obtained by gluing $U_{0}$ to $U_{1}$ with $f_{t}^{-1}$. Since $f_{t}(z)=z+2 t / z+O\left(t^{2}\right)$, the corresponding deformation is given by $\delta(C, v)$ where $v=v_{01}=-(2 / z)(d / d z)=-1 / \omega$. Applying Theorem 2.1 and the residue theorem, we obtain equation (2.9).

The traditional Schiffer variation. It is also common to define the Schiffer variation by simply collapsing the circle $|z|=t$ to a horizontal slit to obtain $X_{t}$. This definition gives the variation

$$
\mu_{p}=\left.\frac{d X_{t}}{d t}\right|_{t=0}=\operatorname{Sch}(z d z, p)=\frac{1}{2} \operatorname{Sch}(\omega, p) .
$$

If we write $q$ locally near $p$ as $q(z) d z^{2}$, then $\left\langle\mu_{p}, q\right\rangle=\pi q(0)$ by (2.9). A similar calculation appears in $[\mathrm{Ga}, \S 5]$. 
The definition we have chosen, which begins and ends with slits, is better adapted to the geometry of holomorphic 1-forms, as we will see in $\S 3$.

The size of deformations. We conclude with some estimates on the size of folding and twisting deformations, using (2.2). The first estimate shows the Schiffer variation at the center of a large disk is small.

Theorem 2.3. Suppose $\omega=z d z$ in a local chart $U \cong \Delta(r)$ on $X$, and $z(p)=0$. Then the Schiffer variation satisfies

$$
\|\operatorname{Sch}(\omega, p)\|_{T} \leq 1 / r^{2}
$$

Proof. For any unit norm $q \in Q(X)$ given locally by $q=q(z) d z^{2}$, we have

$$
|\langle\operatorname{Sch}(\omega, p), q\rangle|=\pi|q(0)| \leq \pi\left(\pi r^{2}\right)^{-1} \int_{\Delta(r)}|q| \leq r^{-2} \int_{X}|q|=r^{-2}
$$

To make an estimate for twists, suppose $X$ contains an annulus given in local coordinates by

$$
A=\{z: 0<\operatorname{Im}(z)<h\} /\langle z \mapsto z+c\rangle
$$

Then $A$ is a right cylinder of height $h$ and circumference $c$ in the metric $|d z|$; hence $\bmod (A)=h / c$. Let $C \subset A$ be a horizontal closed geodesic, oriented so $L(C)=$ $\int_{C} d z=c$, and let $v=d / d z$.

Theorem 2.4. The unit speed twist about the core curve $C$ of A satisfies

$$
\|\delta(C, v)\|_{T} \leq \frac{1}{2 \bmod (A) L(C)} .
$$

Proof. Locally writing $q=q(z) d z^{2}$, we have

$$
|\langle\delta(C, v), q\rangle|=\frac{1}{h} \int_{0}^{h} \frac{1}{2}\left|\int_{0}^{c} q(x+i y) d x\right| d y \leq(2 h)^{-1} \int_{A}|q| \leq(2 h)^{-1}\|q\|_{1} .
$$

Using the collar lemma in hyperbolic geometry, we obtain:

Corollary 2.5. The Fenchel-Nielsen twist around a geodesic C satisfies $\|\operatorname{tw}(C)\|_{T}=$ $O(1)$ when $C$ is short, and $\|\operatorname{tw}(C)\|_{T}=O\left(e^{L(C) / 2}\right)$ when $C$ is long.

These estimates can also be demonstrated by constructing explicit shearing deformations supported in a collar. 


\section{Navigating the space of 1 -forms}

In this section we discuss the moduli space $\Omega \mathcal{M}_{g}$ of holomorphic 1-forms, its strata and its foliations, and the use of Schiffer variations to control the geometry of a 1-form as its relative periods vary. For more on the space $\Omega \mathcal{M}_{g}$, see e.g. [KZ], [Mas].

Moduli spaces, strata and periods. Let $\Omega(X) \cong H^{1,0}(X)$ denote the $g$-dimensional vector space of holomorphic 1-forms on $X$. Assume $\omega \neq 0$, and let $Z(\omega) \subset X$ denote its zero set.

The form $\omega$ determines a natural flat metric $|\omega|$ on $X$ with singularities of negative curvature at its zeros. Geodesics for this metric are straight lines in local coordinates where $\omega=d z$; they may bend at the zeros.

The relative periods of $\omega$ are recorded by the cohomology class

$$
[\omega] \in H^{1}(X, Z(\omega))=H_{1}(X, Z(\omega))^{*}
$$

defined on integral 1-cycles by $\langle[\omega], E\rangle=\int_{E} \omega$.

The set of pairs $(X, \omega)$ with $\omega \neq 0$ forms the moduli space of 1-forms,

$$
\Omega \mathcal{M}_{g} \rightarrow \mathcal{M}_{g} .
$$

This orbifold is a union of strata $\Omega \mathcal{M}_{g}\left(m_{1}, \ldots, m_{s}\right)$ labeled by partitions of $2 g-2$. A form $(X, \omega)$ lies in the given stratum iff $\omega$ has $s$ distinct zeros with multiplicities $m_{1}, \ldots, m_{s}$.

The cohomology groups $H^{1}(X, Z(\omega))$ form a flat bundle over any stratum. Thus we have a locally defined period map

$$
\pi: \Omega \mathcal{M}_{g}\left(m_{i}\right) \rightarrow H^{1}\left(X_{0}, Z\left(\omega_{0}\right)\right) / \operatorname{Aut}\left(X_{0}, \omega_{0}\right)
$$

given by $\pi(X, \omega)=[\omega] \in H^{1}(X, Z(\omega)) \cong H^{1}\left(X_{0}, Z\left(\omega_{0}\right)\right)$. By [V] and [MS, Lemma $1.1]$, the period map is a holomorphic local homeomorphism of orbifolds.

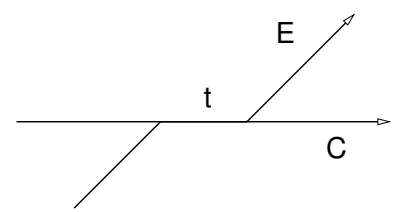

Fig. 3. A right twist on $C$ adds $t$ to the period of $E$.

Twists. In a local orbifold chart, the tangent space to the stratum of $\Omega \mathcal{M}_{g}$ through $(X, \omega)$ can be naturally identified with $H_{1}(X, Z(\omega))^{*}$. The orbifold structure is recorded by the action of the finite $\operatorname{group} \operatorname{Aut}(X, \omega)$. Similarly, the tangent space to $\mathcal{M}_{g}$ at $X$ in an orbifold chart is given by $H^{1}(X ; \Theta)$, equipped with the action of $\operatorname{Aut}(X)$. The projection $\Omega \mathcal{M}_{g} \rightarrow \mathcal{M}_{g}$ is a smooth map of orbifolds, compatible with the inclusion $\operatorname{Aut}(X, \omega) \subset \operatorname{Aut}(X)$. 
Any smooth, simple loop $C$ in $X-Z(\omega)$ determines an element in the dual of $H_{1}(X, Z(\omega))$ by

$$
\langle\operatorname{tw}(C), E\rangle=C \cdot E ;
$$

thus $C$ also determines a tangent vector tw $(C)$ to $\Omega \mathcal{M}_{g}$ at $(X, \omega)$, in the sense of orbifolds. The vector $\operatorname{tw}(C)$ depends only on the homology class $[C] \in H_{1}(X-Z(\omega))$.

For a more geometric picture, consider the case where $C$ is a closed horizontal geodesic on $(X,|\omega|)$. Then we can cut $X$ open along $C$, twist distance $t$ to the right and reglue to obtain a new holomorphic form $\left(X_{t}, \omega_{t}\right)$. The periods of the twisted form $\omega_{t}$ are given by

$$
\int_{E} \omega_{t}=\int_{E} \omega_{0}+t C \cdot E,
$$

as can be seen from Figure 3. A similar twist can be carried out along an oriented closed geodesic of slope $\theta$, and we find:

Twisting to the right along $C$ defines an integral curve $\left(X_{t}, \omega_{t}\right)$ of the vector field

$e^{i \theta} \operatorname{tw}(C)$ for all $t \in \mathbb{R}$.

To treat the case of general $C$, let $\Theta^{\omega} \subset \Theta$ denote the sheaf of translation vector fields on $X$. By definition, a section of $\Theta^{\omega}$ is a holomorphic vector field $v$ satisfying $d(\omega(v))=0$. This condition means that $v=a(d / d z)$ in local coordinates where $\omega=d z$, and $v=0$ at the zeros of $\omega$. The stalks of $\Theta^{\omega}$ are 1-dimensional except over the zeros of $\omega$, where they are 0 -dimensional.

There is a unique translation vector field $v$ defined near $C$ satisfying $\omega(v)=1$. Thus $C$ and $\omega$ determine a cocycle

$$
\Delta(C, v) \in H^{1}\left(X ; \Theta^{\omega}\right),
$$

lifting $\delta(C, v) \in H^{1}(X ; \Theta)$ to a deformation of the translation structure defined by $\omega$. This cocycle also represents $\operatorname{tw}(C)$, which shows:

Theorem 3.1. The projection $\Omega \mathcal{M}_{g} \rightarrow \mathcal{M}_{g}$ sends the tangent vector $\operatorname{tw}(C)=$ $\Delta(C, 1 / \omega)$ to $\delta(C, 1 / \omega)$.

Corollary 3.2. The derivative of the projection map $\pi: \Omega \mathcal{M}_{g}\left(m_{i}\right) \rightarrow \mathcal{M}_{g}$ satisfies

$$
\left\langle\pi_{*}(\operatorname{tw}(C)), q\right\rangle=\frac{i}{2} \int_{C} q \omega
$$

for any $[C] \in H_{1}(X-Z(\omega))$ and $q \in Q(X)$.

This follows from Theorem 2.1.

Remark. There is a natural isomorphism

$$
H^{1}\left(X ; \Theta^{\omega}\right) \cong H^{1}(X, Z(\omega)) \cong T_{(X, \omega)} \Omega \mathcal{M}_{g}\left(m_{i}\right)
$$

coming from the exact sequence of sheaves

$$
0 \rightarrow \Theta^{\omega} \rightarrow \mathbb{C} \rightarrow \bigoplus_{P \in Z(\omega)} \mathbb{C}_{P} \rightarrow 0 .
$$


(The second arrow is given by $v \mapsto v / \omega$.) In these terms, the derivative of the projection $\Omega \mathcal{M}_{g}\left(m_{i}\right) \rightarrow \mathcal{M}_{g}$ can also be identified with the natural map $H^{1}\left(X ; \Theta^{\omega}\right) \rightarrow H^{1}(X ; \Theta)$.

The Schiffer variation revisited. In Section 2 we saw that a simple zero $p$ of $\omega$ determines a deformation $\operatorname{Sch}(\omega, p)$ of $X$ supported near $p$. This construction also lifts to a deformation of the 1-form $(X, \omega)$.

Indeed, by $(2.8)$ the data $(p, \omega)$ determines a path of 1 -forms $\left(X_{t}, \omega_{t}\right)$, as well as holomorphic maps

$$
f_{t}: X-A_{t} \rightarrow X_{t}-B_{t}
$$

such that $f_{t}^{*} \omega_{t}=\omega$. Geometrically, the new form $\left(X_{t}, \omega_{t}\right)$ is obtained from $(X, \omega)$ by opening the vertical arc $A_{t}$ to obtain a square with sides of length $t$, and then folding its sides together to form a horizontal $\operatorname{arc} B_{t}$ (see Figure 4).

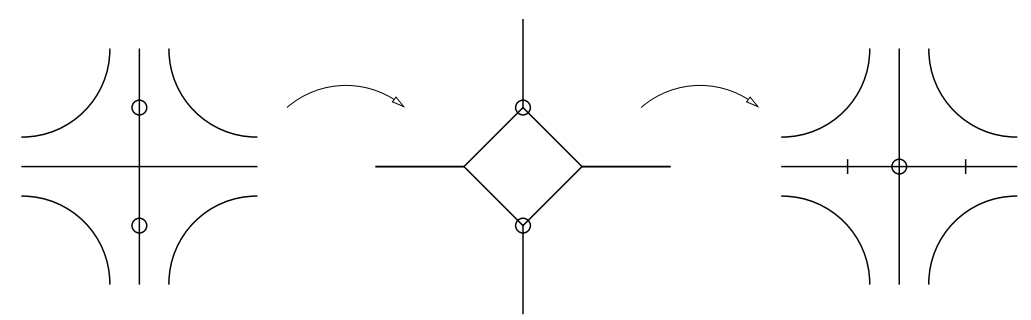

Fig. 4. Folding at a zero of $\omega$.

There is an analogous folding operation when $p$ is a zero of order $n>1$. In this case $A_{t}$ is a union of $n+1$ geodesic rays $\left[p, e_{i}\right]$ with $\int_{p}^{e_{i}} \omega_{0}=t$. Cutting along $A_{t}$ yields a $2 n+2$-gon, which we refold to obtain $B_{t}$.

Let $C_{p} \subset X-Z(\omega)$ be a positively oriented loop around $p$. The following observations will be useful:

- The path $\left(X_{t}, \omega_{t}\right)$ is an integral curve of the vector field $-\operatorname{tw}\left(C_{p}\right)$. (For simple zeros this follows from Theorem 2.2; the generalization to zeros of higher order is straightforward.)

- The path $\left(X_{t}, \omega_{t}\right)$ can be defined for all $t \in[0, T]$, provided $A_{T}$ embeds in $X$.

- Folds at different zeros $p, p^{\prime}, p^{\prime \prime}, \ldots$ can be carried out simultaneously up to time $T$, provided the $\operatorname{arcs} A_{T}, A_{T}^{\prime}, A_{T}^{\prime \prime}, \ldots$ are disjoint.

Saddle connections. A geodesic segment $C=\left[p, p^{\prime}\right]$ on $(X,|\omega|)$ is a saddle connection if $p$ and $p^{\prime}$ are zeros of $\omega$, and there no zeros between them on $C$. (We allow the case $p=p^{\prime}$.) The length $R$ of the shortest saddle connection on $(X, \omega)$ is a useful measure of its 'injectivity radius'; for example, the open balls of radius $R / 2$ about different zeros of $\omega$ are embedded and disjoint. 
The absolute period foliation. The absolute period map is the locally defined submersion of orbifolds

$$
\Omega \mathcal{M}_{g} \supset U \rightarrow H^{1}\left(X_{0}\right) / \operatorname{Aut}\left(X_{0}\right),
$$

which sends a form $(X, \omega)$ with $X$ near $X_{0}$ to the cohomology class $[\omega] \in H^{1}(X) \cong$ $H^{1}\left(X_{0}\right)$. Its fibers form the leaves of the absolute period foliation $\mathcal{A}$.

Since $\Omega(X) \rightarrow H^{1}(X)$ is injective, $\mathcal{A}$ is transverse to the fibers of the projection $\pi: \Omega \mathcal{M}_{g} \rightarrow \mathcal{M}_{g}$. By the same reasoning, $\mathcal{A}$ is transverse to the orbits of the $\mathrm{SL}_{2}(\mathbb{R})$ acting on $\Omega \mathcal{M}_{g}$.

Foliated strata. Let $\mathcal{A}\left(m_{i}\right)$ denote the restriction of $\mathcal{A}$ to a stratum $\Omega \mathcal{M}_{g}\left(m_{i}\right)$. The tangent space to a leaf of $\mathcal{A}\left(m_{i}\right)$ is simply the kernel of the map

$$
H^{1}(X, Z(\omega)) \rightarrow H^{1}(X)
$$

It is generated by the deformations $\operatorname{tw}\left(C_{p}\right), p \in Z(\omega)$, subject to the relation $\sum \operatorname{tw}\left(C_{p}\right)$ $=0$. In particular, the leaves of $\mathcal{A}\left(m_{i}\right)$ have dimension $s-1$. A natural metric along the leaves is given by

$$
\left\|\sum a_{p} \operatorname{tw}\left(C_{p}\right)\right\|_{R}=\max \left|a_{p}-a_{q}\right|
$$

It is convenient to set

$$
\|\operatorname{tw}(C)\|_{R}=\infty
$$

whenever $\operatorname{tw}(C)$ is not tangent to $\mathcal{A}$.

Geometry of $\mathcal{A}$. Let $\mathbb{X}=\mathbb{C}^{s} / \mathbb{C}(1, \ldots, 1)$. For any open disk $U$ in a leaf of $\mathcal{A}\left(m_{i}\right)$, the relative period map $\rho: U \rightarrow \mathbb{X}$ is defined by

$$
\rho(X, \omega)=\left(\int_{q}^{p_{1}} \omega, \ldots, \int_{q}^{p_{s}} \omega\right) .
$$

Here $\left(p_{1}, \ldots, p_{s}\right)$ are the zeros of $\omega$. Since we have taken the quotient of $\mathbb{C}^{s}$ by the constant vector, this map does not depend on $q$. It does depend on the ordering of the zeros and the choice of paths from $q$ to $p_{i}$; but since the absolute periods are constant, changing these choices only translates $\rho(X, \omega)$ by a constant and permutes its coordinates.

Thus the leaves of $\mathcal{A}\left(m_{i}\right)$ are naturally $(G, \mathbb{X})$ manifolds, where $G=\operatorname{Sym}\left(m_{i}\right) \ltimes \mathbb{C}^{s-1}$ and $\operatorname{Sym}\left(m_{i}\right)$ is the group of permutations $\sigma$ satisfying $m_{\sigma i}=m_{i}$. In the case $\left(m_{i}\right)=$ $(g-1, g-1)$, the leaves of $\mathcal{A}\left(m_{i}\right)$ are Riemann surfaces and this $(G, \mathbb{X})$ structure is given by a holomorphic quadratic differential on each leaf. See [Mc3] and [Ba] for examples in genus two, where one obtains a foliated Hilbert surface.

Geodesics. A relative path is a smooth map $\gamma:[a, b] \rightarrow \Omega \mathcal{M}\left(m_{i}\right)$ whose length

$$
L(\gamma)=\int_{a}^{b}\left\|\gamma^{\prime}(t)\right\|_{R} d t
$$

in the metric (3.2) is finite. Our convention (3.3) implies that $\gamma$ lies in a leaf of the absolute period foliation. 
A relative period such as $\int_{p_{1}}^{p_{2}} \omega$ can change by at most $L(\gamma)$ along a relative path. The same is true of the length of the shortest saddle connection.

A geodesic is a path in a stratum such that $\gamma^{\prime}(t) \in H^{1}(X, Z(\omega))$ is locally constant. If $\gamma$ also lies in a leaf of $\mathcal{A}$, we say it is a relative period geodesic.

Theorem 3.3. Let $R$ be the length of the shortest saddle connection on $(X, \omega)$. Then the relative period geodesic $\left(X_{t}, \omega_{t}\right)$ tangent to a unit vector $\sum a_{p} \operatorname{tw}\left(C_{p}\right)$ at $(X, \omega)$ is defined for $|t|<R / 2$. Moreover we have a continuous family of holomorphic embeddings

$$
f_{t}:\left(X-D_{t}, \omega\right) \rightarrow\left(X_{t}, \omega_{t}\right),
$$

where $D_{t}$ is the $|t|$-neighborhood of $Z(\omega)$.

Proof. By the definition (3.2) of a unit vector, we may assume $\left|a_{p}\right| \leq 1$ for all $p$. Then so long as $|t|$ is less than half the length of the shortest saddle connection, the rays of length $t\left|a_{p}\right|$ at different zeros $p$ are disjoint, embedded and lie within distance $t$ of $p$. Thus the folding construction is defined simultaneously at each zero, and it does not change the form outside of $D_{t}$.

Corollary 3.4. Every leaf of $\mathcal{A}\left(m_{i}\right)$ contains an immersed ball of radius $R(X, \omega) / 2$ centered at $(X, \omega)$.

\section{Relative period geodesics}

In this section we use the space of 1-forms on stable curves to analyze the behavior of relative period geodesics in $\Omega \mathcal{M}_{g}$. For more on $\Omega \overline{\mathcal{M}_{g}}$, see e.g. [HM], [Ba, Ch. 5].

Stable curves. A stable curve $Y$ is a connected 1-dimensional complex variety with only nodal singularities, such that its smooth locus $Y^{*}$ has a finite automorphism group.

Let $\Omega(Y)$ denote the space of holomorphic 1-forms on $Y^{*}$ with at worst simple poles, of opposite residues, at any pair of punctures forming a node. The arithmetic genus of $Y$ (so long as it is singular) is given by

$$
g=\operatorname{dim} \Omega(Y)=\sum_{i=1}^{s} g_{i}-s+2|N(Y)|
$$

where $\left(g_{1}, \ldots, g_{s}\right)$ are the genera of the components of $Y^{*}$, and $N(Y) \subset Y$ is the set of nodes. The moduli space $\overline{\mathcal{M}_{g}}$ of stable curves of genus $g$ provides a natural compactification of $\mathcal{M}_{g}$. Moreover, the bundle $\Omega \mathcal{M}_{g}$ extends to $\overline{\mathcal{M}_{g}}$ with fibers $(Y, \eta)$, $0 \neq \eta \in \Omega(Y)$. Thus the corresponding bundle of projective spaces,

$$
\mathbb{P} \Omega \overline{\mathcal{M}_{g}} \rightarrow \overline{\mathcal{M}_{g}}
$$

is compact.

Sketch of the proof. This compactness can be seen analytically as follows. Let $\left(X_{n}, \omega_{n}\right)$ be a sequence of forms in $\Omega \mathcal{M}_{g}$. Pass to a subsequence such that $X_{n}$ converges to a stable 
curve $Y$. Then $X_{n} \rightarrow Y$ geometrically with respect to their hyperbolic metrics $\rho_{n}$ and $\rho$. Scale $\omega_{n}$ so $\left|\omega_{n}\right| / \rho_{n} \leq 1$ in the hyperbolic thick part of $X_{n}$. By compactness of bounded analytic functions, we can assume $\omega_{n}$ converges to a limiting form $\omega$ on the thick part of $Y$.

Now any component of the thin part of $X_{n}$ is isomorphic to an annulus $A\left(r_{n}\right)=\{z$ : $\left.r_{n} \leq|z| \leq 1\right\}$, and $\rho_{n} \asymp|d z| /|z|$ near the boundary of $A\left(r_{n}\right)$. Thus $\omega_{n}=\omega_{n}(z) d z / z$, where $\omega_{n}(z)$ is uniformly bounded in $A_{n}\left(r_{n}\right)$ and converging near $S^{1}$. Assume $r_{n} \rightarrow 0$, so $Y$ acquires a cusp; then $\omega_{n}(z)$ converges to a bounded analytic function $\omega(z)$ with a removable singularity on the punctured disk. Consequently, $\omega=\omega(z) d z / z$ has at worst a simple pole. The condition of opposite residues follows by considering $\int_{|z|=1} \omega_{n}$.

For more details, see e.g. [Wol3].

Compactness and mass. Let $m(X, \omega)=\int_{X}|\omega|^{2}$ denote the total area or mass of a given 1-form. Let $\Omega_{[a, b]} \overline{\mathcal{M}_{g}}$ denote the space of stable forms with $m(X, \omega) \in[a, b]$. (Such forms have no poles. We allow $\omega=0$ if $a=0$.) By Fatou's lemma, if $\left(X_{n}, \omega_{n}\right) \rightarrow(Y, \eta)$ then we have

$$
m(Y, \eta) \leq \liminf m\left(X_{n}, \omega_{n}\right)
$$

thus $\Omega_{[0,1]} \overline{\mathcal{M}_{g}}$ is compact.

However, the locus $\Omega_{1} \overline{\mathcal{M}_{g}}$ where $m(X, \omega)=1$ is not compact. For example, we can find forms with $m\left(X_{n}, \omega_{n}\right)=1$ such that $\left(X_{n}, \omega_{n}\right) \rightarrow(Y, \eta)$ and $Y$ is a union of curves of genus zero; then necessarily $\eta=0$.

Collapsing. A pair of forms $\left(Y_{i}, \eta_{i}\right) \in \Omega \overline{\mathcal{M}}_{g}, i=1,2$, are equivalent if they are isomorphic after removing the components of $Y_{i}^{*}$ where $\eta_{i}$ is identically zero. By collapsing equivalent forms to a single point, we obtain a quotient space $\Omega \overline{\mathcal{M}_{g}} / \sim$.

We now show that relative paths of finite length have well-defined limits, after collapsing.

Theorem 4.1. Let $\gamma(t)=\left(X_{t}, \omega_{t}\right) \in \Omega \mathcal{M}_{g}\left(m_{i}\right)$ be a relative path of finite length, defined for $t \in[0,1)$. Then the limit

$$
(Y, \eta)=\lim _{t \rightarrow 1}\left(X_{t}, \omega_{t}\right)
$$

exists in $\Omega \overline{\mathcal{M}_{g}} / \sim$, and there is no loss of mass:

$$
m(Y, \eta)=\lim _{t \rightarrow 1} m\left(X_{t}, \omega_{t}\right)
$$

Oscillating Riemann surfaces. We begin the discussion of Theorem 4.1 by showing the result is false without collapsing.

Theorem 4.2. There exists a path $\gamma(t)=\left(X_{t}, \omega_{t}\right)$ as above such that $\left[X_{t}\right]$ has no limit in $\overline{\mathcal{M}_{g}}$ as $t \rightarrow 1$. 


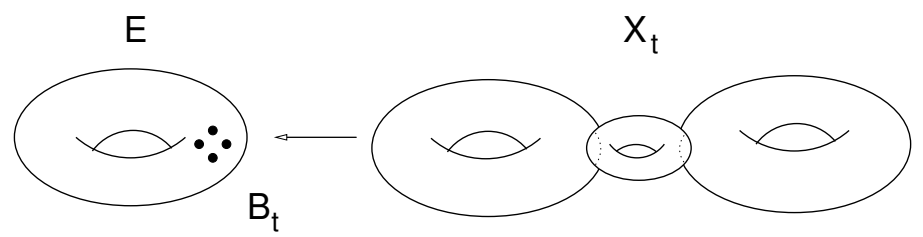

Fig. 5. A degree two branched cover.

Proof. Let $E=\mathbb{C} / \mathbb{Z}[i]$ be the square torus. Let $D \subset E$ be the ball $|z|<1 / 2$, and for each $t \in[0,1)$ let $B_{t} \subset D$ be a set of four distinct points, $p_{i}(t), i=1, \ldots, 4$. Assume $\left|d p_{i} / d t\right|<1 / 2$ and $p_{i}(t) \rightarrow 0$ as $t \rightarrow 1$.

Let $\pi_{t}: X_{t} \rightarrow E$ be the unique degree two covering space branched over $B_{t}$ such that $E-D$ lifts to $X_{t}$. There are two such lifts (see Figure 5). Let $\omega_{t}=\pi_{t}^{*}(d z)$. Then $X_{t}$ has genus three, and $\gamma(t)=\left(X_{t}, \omega_{t}\right)$ is a path in $\Omega \mathcal{M}_{3}(1,1,1,1)$ with constant absolute periods and with $\left\|\gamma^{\prime}(t)\right\|_{R} \leq 1$.

Since the branch locus collapses to a single point as $t \rightarrow 1, X_{t}$ is close to a stable curve made up of two copies of $E$ together with a third elliptic curve $F_{t}$. But the location of $F_{t}$ in $\mathcal{M}_{1}$ depends on the cross-ratio of the points of $B_{t}$. Since we can arrange that this cross-ratio oscillates as $t \rightarrow 1$, we can also arrange that $\left[X_{t}\right]$ has no limit in $\overline{\mathcal{M}_{3}}$.

On the other hand, if we pass to a subsequence $t_{n} \rightarrow 1$ such that $(Y, \eta)=\lim \gamma\left(t_{n}\right)$ exists, then $(Y, \eta)$ consists of two copies of $(E, d z)$ and one additional elliptic curve $(F, \xi)$. The limiting projection $\pi: Y \rightarrow E$ is constant on $F$, so $\xi=0$. (This also follows from the fact that $\lim m\left(X_{t}, \omega_{t}\right)=2=\int_{Y-F}|\eta|^{2}$.) Thus all such limits $(Y, \eta)$ are equivalent.

Conservation of mass. We now turn to the proof of Theorem 4.1. Reparameterizing, we may assume $\left\|\gamma^{\prime}(t)\right\|_{R} \leq 1$. We may also assume $m\left(X_{t}, \omega_{t}\right)=1$ for all $t$ (recall that $\int|\omega|^{2}$ depends only on its absolute periods). Since $\Omega_{[0,1]} \overline{\mathcal{M}_{g}}$ is compact, the forms $\left(X_{t}, \omega_{t}\right)$ have some accumulation point $(Y, \eta)$.

Lemma 4.3. Any accumulation point satisfies $m(Y, \eta)=1$.

Proof. Choose a sequence $t_{n} \rightarrow 1$ such that $\left(X_{n}, \omega_{n}\right)=\gamma\left(t_{n}\right) \rightarrow(Y, \eta)$. Passing to a further subsequence, we can assume that the probability measures $\left|\omega_{n}\right|^{2}$ on $X_{n}$ converge to a measure

$$
|\eta|^{2}+\sum_{p \in N(Y)} a_{p} \delta_{p}
$$

on $Y$, where $a_{p} \geq 0$ and $m(Y, \eta)+\sum a_{p}=1$. Our goal is to show $a_{p}=0$ for all $p$, i.e. no mass escapes to the nodes of $Y$. Note that $\eta$ has no poles, since its mass is finite.

Suppose to the contrary that $a_{p}>0$. Choose any $\epsilon>0$ that is much less than $a_{p}$. Choose a neighborhood $A$ of $p$ in $Y$ consisting of two disks meeting at the node, and small enough that $\int_{\partial A}|\eta|<\epsilon$. (This is possible since $\eta$ is holomorphic at $p$.) Then for all $n \gg 0$, there is a corresponding annulus $A_{n} \subset X_{n}$ such that $\int_{\partial A_{n}}\left|\omega_{n}\right|<\epsilon$.

Since limsup $\int_{A_{n}}\left|\omega_{n}\right|^{2} \geq a_{p}$, we can find a large value $n=N$ and a Euclidean cylin$\operatorname{der} C_{N} \subset\left(A_{N}, \omega_{N}\right)$ such that $\int_{C_{N}}\left|\omega_{N}\right|^{2} \geq a_{p} / 2$. (See Theorem A.2 of the Appendix.) 
Let $h$ and $c$ denote the height and circumference of $C_{N}$. Note that $c$ is the absolute value of the period of $\omega_{N}$ around the core of $C_{N}$. Since this period can also be computed by integrating around a boundary component of $A_{N}$, we have $c<\epsilon$. Thus $h \geq a_{p} /(2 \epsilon) \asymp 1 / \epsilon$.

Shrinking $C_{N}$ slightly, we can assume $C_{N}$ is at distance at least one from $Z\left(\omega_{N}\right)$ in the metric $\left|\omega_{N}\right|$. Since the relative periods of $\left(X_{t}, \omega_{t}\right)$ change at most by one, Theorem 3.3 provides a continuous family of isomorphic embeddings

$$
f_{t}:\left(C_{N}, \omega_{N}\right) \rightarrow\left(X_{t}, \omega_{t}\right),
$$

defined for all $t$. In particular, $\left(X_{0}, \omega_{0}\right)$ contains a Euclidean cylinder of height $h \asymp 1 / \epsilon$. Since the diameter of $\left(X_{0},\left|\omega_{0}\right|\right)$ is bounded, this is a contradiction.

Proof of Theorem 4.1. It remains only to show that as $t \rightarrow 1$, any two accumulation points $\left(Y_{1}, \eta_{1}\right)$ and $\left(Y_{2}, \eta_{2}\right)$ of $\gamma(t)$ are equivalent. Fix $\epsilon>0$. Let $K_{1} \subset Y_{1}^{*}-Z\left(\eta_{1}\right)$ be a compact subsurface with area at least $1-\epsilon$ and boundary of length less than $\epsilon$, in the $\left|\eta_{1}\right|$ metric. Let $\delta>0$ denote the minimum distance between $K_{1}$ and $N\left(Y_{1}\right) \cup Z\left(\eta_{1}\right)$.

We claim $\left(K_{1}, \eta_{1}\right)$ embeds isomorphically in $\left(X_{t}, \omega_{t}\right)$ for all $t$ close enough to one. Indeed, the fact that $\gamma(t)$ accumulates on $\left(Y_{1}, \eta_{1}\right)$ means that we have a sequence $t_{n} \rightarrow 1$ and smooth maps $f_{n}: K_{1} \rightarrow X_{n},\left(X_{n}, \omega_{n}\right)=\gamma\left(t_{n}\right)$, such that

$$
f_{n}^{*} \omega_{n} \rightarrow \eta_{1}
$$

as $C^{\infty}$ forms on $K_{1}$. We also have $d\left(f_{n}\left(K_{1}\right), Z\left(\omega_{n}\right)\right)>\delta / 2$ for all $n$ sufficiently large. By Theorem 3.3, this implies $X_{t}$ contains a slightly distorted copy of $\left(K_{1}, \eta_{1}\right)$ for all $t \in(1-\delta / 4,1)$. Passing to the limit as $n \rightarrow \infty$, we obtain an undistorted copy as well.

Now consider a second sequence $t_{n}^{\prime} \rightarrow 1$ such that $\gamma\left(t_{n}^{\prime}\right) \rightarrow\left(Y_{2}, \eta_{2}\right)$. Then the maps above also yield, in the limit, a holomorphic embedding $\left(K_{1}, \eta_{1}\right) \rightarrow\left(Y_{2}, \eta_{2}\right)$. Letting $\epsilon$ tend to zero, a normal families argument further implies we have an embedding

$$
f: Y_{1}^{*}-Z\left(\eta_{1}\right) \rightarrow Y_{2}^{*}
$$

sending $\eta_{1}$ to $\eta_{2}$. Since both forms have total mass one, the image of $f$ is $Y_{2}^{*}-Z\left(\eta_{2}\right)$. It is routine to check that $f$ extends to the closure of its domain in $Y_{1}$, and thus $\left(Y_{1}, \eta_{1}\right)$ and $\left(Y_{2}, \eta_{2}\right)$ are equivalent.

Examples. Let $\left(E_{i}, \omega_{i}\right)=\left(\mathbb{C} / \Lambda_{i}, d z\right)$ be a pair of forms of genus one. We define $T=$ $\min \left(\lambda \in \Lambda_{1} \cup \Lambda_{2}: \lambda>0\right)$. Then the connected sum

$$
\gamma(t)=\left(X_{t}, \omega_{t}\right)=\left(E_{1}, \omega_{1}\right) \underset{[0, t]}{\#}\left(E_{2}, \omega_{2}\right)
$$

(cf. $[\mathrm{Mc} 2, \S 7])$ gives a relative period geodesic

$$
\gamma:(0, T) \rightarrow \Omega \mathcal{M}_{2}(1,1) .
$$

As $t \rightarrow 0, \gamma(t)$ terminates via the creation of a node; the limit $(Y, \eta)$ is obtained by simply identifying $E_{1}$ and $E_{2}$ at $z=0$.

The behavior as $t \rightarrow T$ is more interesting. If $T \in \Lambda_{1}$ but $T \notin \Lambda_{2}$, then $\left(X_{T}, \omega_{T}\right)$ lies in $\Omega \mathcal{M}_{2}(2)$ and $\gamma(t)$ terminates via a collision of the zeros of $\omega_{t}$. On the other hand, 
if $T$ belongs to both $\Lambda_{1}$ and $\Lambda_{2}$, then we can write these lattices $\Lambda_{i}=\mathbb{Z} T \oplus \mathbb{Z} \tau_{i}$ with $\tau_{i} \in \mathbb{H}$. In this case $\gamma(t)$ terminates at the stable form $(Y, \eta)$ obtained from

$$
(E, \omega)=(\mathbb{C} / \Lambda, d z), \quad \Lambda=\mathbb{Z} T \oplus \mathbb{Z}\left(\tau_{1}+\tau_{2}\right),
$$

by gluing $z=0$ to $z=\tau_{1}$ to form a node.

Finally, if $T=\infty$ we obtain a complete geodesic ray in $\Omega \mathcal{M}_{2}$. It would be interesting to understand the asymptotic distribution of this ray and other period geodesics, including those transverse to $\mathcal{A}$.

\section{Appendix: Flat metrics and cylinders}

In this section we collect some basic length-area bounds for disks and cylinders. These bounds are used in $\S 4$.

Proposition A.1. Let $\omega$ be a holomorphic 1-form on a disk $D$. Then the area of $D$ in the metric $|\omega|$ is controlled by the length of its boundary:

$$
\int_{X}|\omega|^{2} \leq \frac{1}{4}\left(\int_{\partial X}|\omega|\right)^{2}
$$

Note that $\omega$ is allowed to have zeros in $D$.

Proof. Let $L=\int_{\partial X}|\omega|$ and let $\omega=d f$. If we normalize so that $f(p)=0$ for some $p \in \partial D$, then we have $\max _{\partial D}|f| \leq L / 2$. Thus

$$
\int_{X}|\omega|^{2}=\frac{i}{2} \int_{X} d(f \bar{\omega}) \leq \frac{1}{2} \int_{\partial D}|f \omega| \leq \frac{L^{2}}{4} .
$$

The Euclidean cylinder $(C, d z)$ of height $h$ and circumference $c$ is given by

$$
C=\{0<\operatorname{Im}(z)<h\} /\langle z \mapsto z+h\rangle .
$$

Theorem A.2. Let $(A, \omega)$ be an annulus equipped with a holomorphic 1-form of total area one such that $L=\int_{\partial A}|\omega|$ is small. Then A contains a Euclidean cylinder of nearly the same area. More precisely, there exists an inclusion of a Euclidean cylinder $(C, d z)$ into $(A, \omega)$ such that

- $\omega \mid C=e^{i \theta} d z$;

- $\int_{A-C}|\omega|^{2} \leq 1-O\left(L^{2}\right) ;$ and

- the inclusion gives an isomorphism $\pi_{1}(C) \cong \pi_{1}(A)$.

Proof. First suppose $\omega=d f$ is exact. Normalize so that $f: A \rightarrow \mathbb{C}$ vanishes at some point on $\partial A$. By assumption, the length of $f(\partial A)$ is $O(L)$. By the open mapping theorem, $f(\partial A)$ is either a connected set or it consists of two immersed loops in $\mathbb{C}$, one of which 


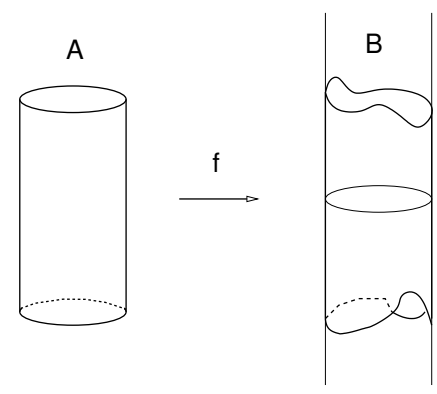

Fig. 6

encloses the other. In either case we find $|f|=O(L)$ on $\partial A$. A variant of (A.1) then implies $1=\int_{A}|\omega|^{2}=O\left(L^{2}\right)$. Since $L$ is small, we may assume this case does not occur.

Now let $c=\int_{\gamma} \omega$ be the period of $\omega$, where $\gamma$ generates $\pi_{1}(A)$. We may assume $c>0$, and certainly $c<L$. Let $B$ be the infinite Euclidean cylinder $\mathbb{C} /\langle z \mapsto z+c\rangle$, equipped with the metric $d z$.

By integrating $\omega$ we obtain a map $f: A \rightarrow B$, inducing an isomorphism $\pi_{1}(A) \cong$ $\pi_{1}(B)$, such that $\omega=f^{*}(d z)$. (See Figure 6.) The image of $\partial A$ under $f$ gives a pair of immersed loops $\gamma_{1}, \gamma_{2} \subset B$ each of length less than $L$ in the Euclidean metric $|d z|$.

We may assume the minimum of $\operatorname{Im} f(z)$ on $A$ is 0 ; let its maximum be $h$. By considering the preimage of the vertical segment $i[0, h] \subset B$, we obtain an arc $\alpha$ joining the ends of $A$ with $\int_{\alpha}|\omega| \leq h$. Cutting along $\alpha$ to obtain a disk and applying Proposition A.1, we find $1=\int_{A}|\omega|^{2} \leq h^{2}+O\left(h L+L^{2}\right)$. Since $L$ is small, we may assume $h>1 / 2 \gg L$. Consequently,

$$
C=\{z: \operatorname{Im}(z) \in[L, h-L]\} \subset B
$$

is a nontrivial cylinder disjoint from $\partial A$.

Let $C^{\prime}=f^{-1}(C)$. Since $f: C^{\prime} \rightarrow C$ is proper, every component of $C^{\prime}$ has at least two boundary components. But every component of $A-C^{\prime}$ must meet $\partial A$, by the maximum principle for $\pm \operatorname{Im}(f)$. Thus $C^{\prime}$ consists of a single annulus and $f: C^{\prime} \rightarrow C$ is an isomorphism. Using Proposition A.1 again, we find the integral of $|\omega|^{2}$ over the preimage of $B-C$ is $O\left(L^{2}\right)$, and hence $f^{-1}: C \rightarrow A$ gives the required embedded cylinder.

Remark. The same result can be proved more analytically by normalizing so $A=\{z$ : $1<|z|<R\}$ and then studying $\omega=\sum a_{n} z^{n} d z$ as an element of unit norm in $L^{2}(A)$.

Acknowledgments. This research was supported in part by the NSF, the MPI and the Humboldt Foundation.

\section{References}

[Ah] Ahlfors, L.: Conformal Invariants: Topics in Geometric Function Theory. McGraw-Hill (1973) Zbl 0272.30012 MR 0357743 
[ACG] Arbarello, E., Cornalba, M., Griffiths, P. A.: Geometry of Algebraic Curves. Vol. 2, Springer (2011) Zbl 1235.14002 MR 2807457

[Ba] Bainbridge, M.: Euler characteristics of Teichmüller curves in genus two. Geom. Topol. 11, 1887-2073 (2007) Zbl 1131.32007 MR 2350471

[D] DeMarco, L.: The conformal geometry of billiards. Bull. Amer. Math. Soc. 48, 33-52 (2011) Zbl 1225.37049 MR 2738905

[Ga] Gardiner, F.: Schiffer's interior variation and quasiconformal mapping. Duke Math. J. 42, 371-380 (1975) Zbl 0347.30017 MR 0382637

[GL] Gardiner, F. P., Lakic, N.: Quasiconformal Teichmüller Theory. Amer. Math. Soc. (2000) Zbl 0949.30002 MR 1730906

[HM] Harris, J., Morrison, I.: Moduli of Curves. Springer (1998) Zbl 0913.14005 MR 1631825

[IT] Imayoshi, Y., Taniguchi, M.: An Introduction to Teichmüller Spaces. Springer (1992) Zbl 0754.30001 MR 1215481

[Ker] Kerckhoff, S.: The Nielsen realization problem. Ann. of Math. 177, 235-265 (1983) Zbl 0528.57008 MR 0690845

[KZ] Kontsevich, M., Zorich, A.: Connected components of the moduli spaces of Abelian differentials with prescribed singularities. Invent. Math. 153, 631-678 (2003) Zbl 1087.32010 MR 2000471

[Mas] Masur, H.: Ergodic theory of translation surfaces. In: Handbook of Dynamical Systems, Vol. 1B, Elsevier, 527-547 (2006) Zbl 1130.37313 MR 2186247

[MS] Masur, H., Smillie, J.: Hausdorff dimension of sets of nonergodic measured foliations. Ann. of Math. 134, 455-543 (1991) Zbl 0774.58024 MR 1135877

[Mc1] McMullen, C.: Complex earthquakes and Teichmüller theory. J. Amer. Math. Soc. 11, 283320 (1998) Zbl 0890.30031 MR 1478844

[Mc2] McMullen, C.: Dynamics of $\mathrm{SL}_{2}(\mathbb{R})$ over moduli space in genus two. Ann. of Math. 165, 397-456 (2007) Zbl 1131.14027 MR 2299738

[Mc3] McMullen, C.: Foliations of Hilbert modular surfaces. Amer. J. Math. 129, 183-215 (2007) Zbl 1154.14020 MR 2288740

[Mc4] McMullen, C.: Moduli spaces of isoperiodic forms on Riemann surfaces. Preprint (2012)

[Mo] Möller, M.: Affine groups of flat surfaces. In: A. Papadopoulos (ed.), Handbook of Teichmüller Theory, Vol. II, Eur. Math. Soc., 369-387 (2009) Zbl 1179.30047 MR 2497782

[Sch] Schiffer, M.: A method of variation within the family of simple functions. Proc. London Math. Soc. 44, 432-449 (1938) Zbl 0019.22201

[SS] Schiffer, M., Spencer, D. C.: Functionals of Finite Riemann Surfaces. Princeton Univ. Press (1954) Zbl 0059.06901 MR 0065652

[Th] Thurston, W. P.: Earthquakes in two-dimensional hyperbolic geometry. In: D. B. Epstein (ed.), Low-Dimensional Topology and Kleinian Groups, Cambridge Univ. Press, 91-112 (1987) Zbl 0628.57009 MR 0903860

[V] Veech, W.: Moduli spaces of quadratic differentials. J. Anal. Math. 55, 117-171 (1990) Zbl 0722.30032 MR 1094714

[Wol1] Wolpert, S.: The Fenchel-Nielsen deformation. Ann. of Math. 115, 501-528 (1982) Zbl 0496.30039 MR 0657237

[Wol2] Wolpert, S.: Cut-and-paste deformations of Riemann surfaces. Ann. Acad. Sci. Fenn. Ser. A I Math. 13, 401-413 (1988) Zbl 0675.30035 MR 0994473

[Wol3] Wolpert, S.: Families of Riemann Surfaces and Weil-Petersson Geometry. Amer. Math. Soc. (2009) 